\title{
吡啶联噁唑酰胺类化合物的设计、合成及杀菌活性
}

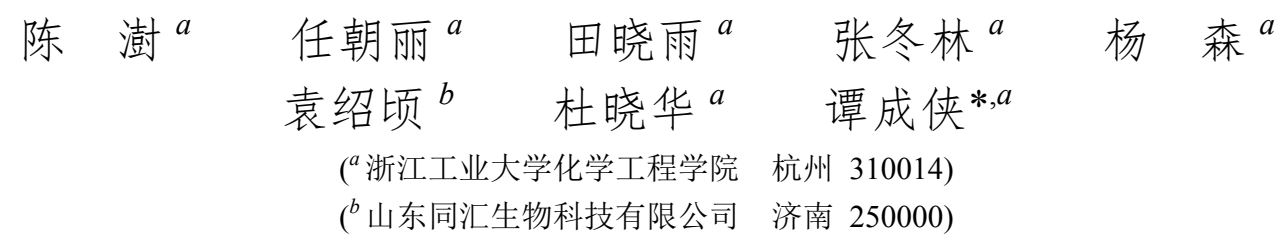

\begin{abstract}
摘要 以吡啶-2-甲醛为起始原料, 经环合、氧化、取代、水解、氯化和胺解反应, 采用中间体衍生化法合成了 13 个新 型的吡啶联噁唑酰胺类化合物, 其结构经 ${ }^{1} \mathrm{H} \mathrm{NMR}$ 和高分辨质谱表征. 初步的杀菌活性测试结果显示, 在 $100 \mathrm{mg} / \mathrm{L}$ 浓 度下有 7 个化合物对黄瓜灰霉病菌(Botrytis cinerea)的抑制率为 100\%, 其中 N-4-氟苯基-2-(吡啶-2-基)-噁唑-4-甲酰胺 (7b)对水稻纹枯病菌(Rhizoctonia solani) 的抑制率也为 $100 \%$, 进一步的杀菌活性测试结果表明, 部分目标化合物杀菌活 性优于嘧菌酯, 值得进一步研究.
\end{abstract}

关键词 啞唑; 酰胺; 吡啶; 杀菌活性

\section{Design, Synthesis and Fungicidal Activities of Pyridyl Oxazoamide Compounds}

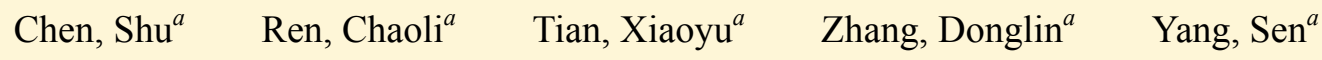 \\ Yuan, Shaoqing $^{b} \quad$ Du, Xiaohua ${ }^{a} \quad$ Tan, Chengxia $^{*, a}$ \\ ( ${ }^{a}$ College of Chemical Engineering, Zhejiang University of Technology, Hangzhou 310014) \\ ( ${ }^{b}$ Shandong Tonghui Biotechnology Co., Ltd, Jinan 250000)
}

\begin{abstract}
Thirteen novel pyridyl oxazoamide compounds were synthesized using pyridine-2-formaldehyde as the starting material through cyclization, oxidation, substitution, hydrolysis, chlorination and ammonolysis reactions via the disclosed intermediate derivatization method (IDM). The structures of title compounds were characterized by ${ }^{1} \mathrm{H}$ NMR and HRMS. Preliminary bioassay results indicated that at $100 \mathrm{mg} / \mathrm{L}, 7$ compounds exhibited $100 \%$ fungicidal activities against Botrytis ciner$e a$, and $N$-(4-fluorophenyl)-2-(pyridin-2-yl)oxazole-4-carboxamide (7b) exhibited 100\% fungicidal activities against $R h i$ zoctonia solani. Further fungicidal activity test results suggested that some of the target compounds had better fungicidal activity than azoxystrobin, which was worthy of further study.
\end{abstract}

Keywords oxazole; amide; pyridine; fungicide activity

随着社会的发展，人们对于农药的毒理和环境影响 等方面有了更高的要求，因此新型农药的研发必不可 少. 酰胺类化合物具有理想的生物活性，被广泛应用于 杀虫、杀菌以及除草等方面 ${ }^{[1-2]}$. 同时在绿色农药创制过 程中，杂环类农药具有活性高及选择性好等特点，成为 农药先导化合物开发热点之一 ${ }^{[3-7]}$, 并占据着十分重要 的地位. 自 19 世纪开始, 相继开发出了不少具有高活性 的含杂环结构的酰胺类杀菌类药物 (图 1) ${ }^{[8-11]}$. 含氮杂环 化合物因其结构形式多样以及良好的生物活性在农药
领域中被广泛应用. 其中, 吡啶类衍生物 ${ }^{[12-17]}$ 与噁唑类 衍生物 ${ }^{[18-23]}$ (图 2)作为重要的含氮杂环化合物也备受关 注.

本工作以氯虫酰胺为先导化合物, 参考噁唑类衍生 物的苯环联噁唑结构, 利用生物电子等排原理, 得到吡 啶联噁唑结构, 再引入苯甲酰胺结构, 最终设计合成一 系列吡啶联噁唑酰胺类化合物, 设计思路如 Scheme 1 . 并且对合成的目标产物进行了结构表征和杀菌活性测 定，活性测定结果表明大部分目标化合物显示出较好的

* Corresponding author. E-mail: tanchengxia@zjut.edu.cn

Received July 3, 2020; revised September 23, 2020; published online October 10, 2020.

Project supported by the Collaborative Innovation Center of Zhejiang Province Green Pesticide.

浙江省绿色农药 2011 协同创新中心开放基金资助项目. 
<smiles>Cc1nn(C)c(Cl)c1C(=O)Nc1cccc2c1C(C)OC2(C)C</smiles><smiles>O=C1C=C(NC(=O)c2cccnc2Cl)C(c2ccc(Cl)cc2)=C1</smiles><smiles>Cn1cc(C(=O)Nc2ccccc2-c2cc(F)c(F)c(F)c2)c(C(F)F)n1</smiles>

Fluxapyroxad

图 1 呋吡菌胺、啶酰菌胺和氟唑菌酰胺的化学结构

Figure 1 Chemical structures of furametpyr, boscalid and fluxapyroxad<smiles>[R]COc1c(OC)ccc(-c2cc(CNC(=O)c3cn(C)nc3C(F)F)on2)c1OC</smiles><smiles>[R]OCOC(=O)/C(C#N)=C(/NCc1cccc([R])c1OCc1coc(-c2cccc([R])c2)n1)SC</smiles>

图 2 啞唑类衍生物的化学结构

Figure 2 Chemical structures of oxazole derivatives 杀菌活性, 当浓度为 $100 \mathrm{mg} / \mathrm{L}$ 时化合物 $7 \mathbf{a} \sim 7 \mathbf{7} 、 7 \mathbf{g} \sim$ $7 \mathbf{m}$ 对黄瓜灰霉病菌的抑制率达 90\% 100\%. 其合成路 线如 Scheme 2 所示.

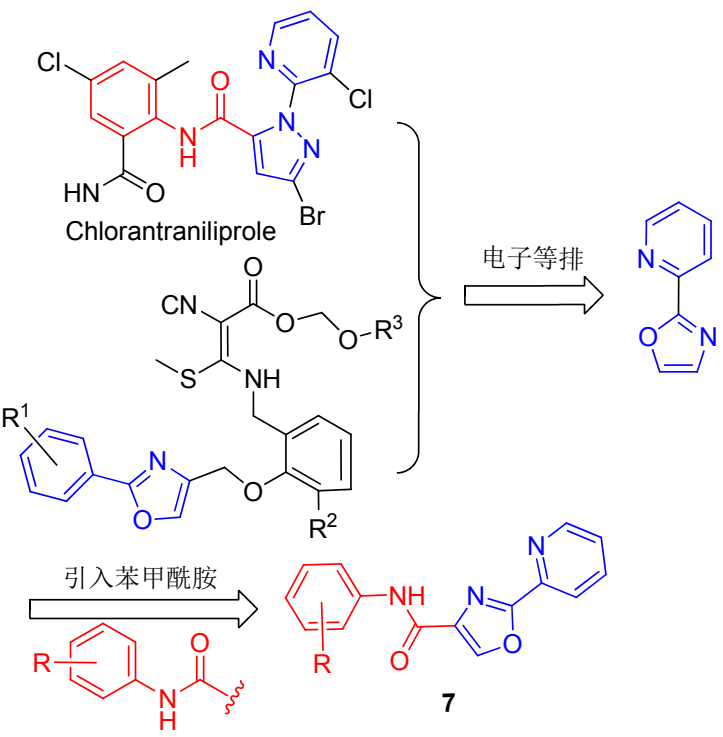

图式 1 目标化合物 7 的分子设计示意图

Scheme 1 Design strategy of the target compounds 7

\section{1 结果与讨论}

\section{1 中间体 4 的合成}

在化合物合成中，以中间体 4 为研究对象，尝试了
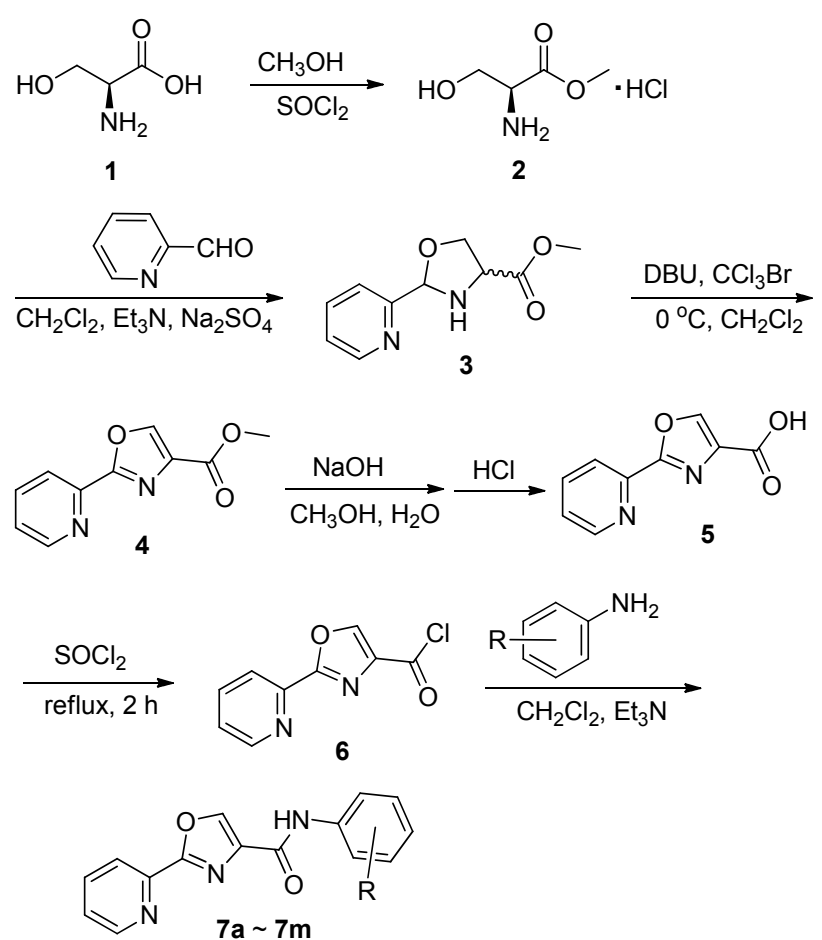

$R=2-F, 4-F, 2,4-F_{2}, 2,6-F_{2}$, etc.

图式 2 目标化合物 $7 \mathrm{a} \sim 7 \mathrm{~m}$ 的合成路线

Scheme 2 Synthetic route of compounds $7 \mathrm{a} \sim 7 \mathrm{~m}$

不同的氧化剂对中间体 4 收率的影响．由表 1 可以看出, 采用三氯溴甲烷和 1,8-二氮杂二环十一碳-7-烯(DBU)作 为氧化剂在室温反应下是制备中间体 $\mathbf{4}$ 的较佳方法，中 间体 4 的收率为 $68 \%$. 在氧化过程中，如果氧化剂用量 不足可能导致噁唑烷结构氧化不彻底产生大量副产物, 经过尝试最终氧化剂与中间体 3 最佳物质的量之比为 6：1. 该方法不仅操作简便，而且后续分离比较容易.

表 1 不同反应条件对中间体 4 合成收率的影响 Table 1 Effects of reaction conditions on the synthesis of compound 4

\begin{tabular}{clc}
\hline Entry & \multicolumn{1}{c}{ Oxidant } & Yield/\% \\
\hline 1 & $\mathrm{~K}_{2} \mathrm{~S}_{2} \mathrm{O}_{8} / \mathrm{H}_{2} \mathrm{SO}_{4}$ & 24 \\
2 & $\mathrm{H}_{2} \mathrm{O}_{2} / \mathrm{H}_{2} \mathrm{SO}_{4}$ & 12 \\
3 & $\mathrm{CCl}_{3} \mathrm{Br} / \mathrm{DBU}$ & 68 \\
\hline
\end{tabular}

\section{2 化合物的波谱分析}

以目标化合物 $7 \mathbf{a}$ 的核磁氢谱为例进行解析. 在 $\delta$ 9.92 处的单峰为 $\mathrm{CONH}$ 上胺基氢的吸收峰, 在 $\delta 8.98$ 处 的单峰为噁唑环上一个氢的吸收峰, 在 $\delta 8.77$ 处的双重 峰为吡啶环上离氮最近的碳上一个氢的吸收峰, 在 $\delta$ 8.22 的双重峰为吡啶环上与噁唑最近的碳上一个氢的 吸收峰, 在 $\delta 8.07 \sim 7.62$ 的多重峰为吡啶环上的两个氢 的吸收峰, 在 $\delta 7.81$ 的三重峰为苯环离酰胺基最近的碳 上的一个氢的吸收峰, 在 $\delta 7.35 \sim 7.27$ 间的多重峰为苯 
环上其余三个氢的吸收峰.

\section{3 化合物的杀菌活性}

目标化合物 7a $\sim \mathbf{7 m}$ 对黄瓜灰霉病菌 (B. cinerea) 与 水稻纹枯病菌 (R. solani) 的杀菌活性测试结果如表 2 所 示. 在 $100 \mathrm{mg} / \mathrm{L}$ 浓度下, 目标化合物对黄瓜灰霉病菌有 较高的抑制效果, 其中化合物 $7 \mathbf{a} \sim 7 \mathrm{e} 、 7 \mathbf{j}$ 以及 $7 \mathbf{k}$ 对黄 瓜灰霉病菌的抑制率为 $100 \%$, 化合物 $7 \mathbf{g} \sim 7 \mathbf{7} 、 71$ 以及 $7 \mathrm{~m}$ 对黄瓜灰霉病菌的抑制率为 $90 \%$, 化合物 7f 对黄瓜 灰霉病菌的抑制率为 $85 \%$. 同时, 大部分目标化合物对 水稻纹枯病菌也有较高的抑制效果, 其中化合物 $7 \mathbf{b}$ 对 水稻纹枯病菌的抑制率为 $100 \%$, 化合物 7a、7e、7 k 对 水稻纹枯病菌的抑制率为 $90 \%$ ，化合物 7c、7d、7g、7h、 7i、7l 对水稻纹枯病菌的抑制率为 $80 \%$, 化合物 $7 \mathbf{j} 、 7 \mathbf{m}$ 对水稻纹枯病菌的抑制率为 $70 \%$. 根据表 2 数据分析可 以看出, 苯环上被取代时, 氟取代的抑制率高于氯取代 以及溴取代的抑制率，甲基取代的抑制率高于叔丁基取 代的抑制率.

因目标化合物 7a $\sim 7 \mathbf{m}$ 在 $100 \mathrm{mg} / \mathrm{L}$ 浓度下表现出理 想杀菌的活性, 对其进行了复篎(表 3). 从表 3 可知, 在 $50 \mathrm{mg} / \mathrm{L}$ 浓度下，化合物 7c、7d、7e、7 $、 7 \mathbf{k} 、 71$ 对黄 瓜灰霉病菌的抑制率均为 $62.06 \% \sim 67.35 \%$, 活性均优 于嘧菌酯, 化合物 7c、7g、7h 对水稻纹枯病菌的抑制率 达 $66.77 \% \sim 79.12 \%$, 活性优于嘧菌酯. 将其中部分目标 化合物与嘧菌酯进行活性对照得到表 4. 根据表 3 数据 分析可以看出在 $50 \mathrm{mg} / \mathrm{L}$ 浓度下, 苯环上取代基为氟和 甲基时, 对于黄瓜灰霉病菌, 其二取代的抑制率高于单 取代. 对于水稻纹枯病菌, 苯环上为二氟取代的化合物 的抑制率高于单氟取代的化合物.
表 $2100 \mathrm{mg} / \mathrm{L}$ 浓度下目标化合物 $7 \mathbf{a} \sim 7 \mathbf{m}$ 的杀菌活性(抑制 率 $/ \%$ )

Table 2 Fungicidal activities (inhibition rate/\%) of compounds $\mathbf{7 a} \sim 7 \mathbf{m}$ at $100 \mathrm{mg} / \mathrm{L}$

\begin{tabular}{clcc}
\hline Compd. & \multicolumn{1}{c}{$\mathrm{R}$} & $\begin{array}{c}\text { 黄瓜灰霉病菌 } \\
(\text { B. cinerea })\end{array}$ & $\begin{array}{c}\text { 水稻纹枯病菌 } \\
\text { (R. solani })\end{array}$ \\
\hline $\mathbf{7 a}$ & $2-\mathrm{F}$ & 100 & 90 \\
$\mathbf{7 b}$ & $4-\mathrm{F}$ & 100 & 100 \\
$\mathbf{7 c}$ & $2,4-\mathrm{F}_{2}$ & 100 & 80 \\
$\mathbf{7 d}$ & $2,6-\mathrm{F}_{2}$ & 100 & 80 \\
$\mathbf{7 e}$ & $3-\mathrm{Cl}$ & 100 & 90 \\
$\mathbf{7 f}$ & $3,5-\mathrm{Cl}_{2}$ & 85 & 60 \\
$\mathbf{7 g}$ & $3-\mathrm{Cl}-2-\mathrm{CH}_{3}$ & 90 & 80 \\
$\mathbf{7 h}$ & $3-\mathrm{Br}_{7}$ & 90 & 80 \\
$\mathbf{7 i}$ & $3-\mathrm{CF}_{3}$ & 90 & 80 \\
$\mathbf{7 j}$ & $4-\mathrm{CH}_{3}$ & 100 & 70 \\
$\mathbf{7 k}$ & $2,4-\left(\mathrm{CH}_{3}\right)_{2}$ & 100 & 90 \\
$\mathbf{7 1}$ & $4-\mathrm{OC}_{2} \mathrm{H}_{5}$ & 90 & 80 \\
$\mathbf{7 m}$ & $4-$ tert $-\mathrm{C}_{4} \mathrm{H}_{9}$ & 90 & 70 \\
嘧菌酯 & & 100 & 100 \\
\hline
\end{tabular}

\section{2 结论}

以吡啶-2-甲醛为起始原料, 经环合、氧化、取代、 水解、氯化和胺解反应合成了 13 个新型的吡啶联噁唑 酰胺类化合物. 其初步的杀菌活性测试结果显示, 部分 目标化合物在 $100 \mathrm{mg} / \mathrm{L}$ 下对黄瓜灰霉病菌的抑制率为 $100 \%$, 大部分目标化合物在 $100 \mathrm{mg} / \mathrm{L}$ 下对黄瓜灰霉病 菌和水稻纹枯病菌的抑制率较高, 其中目标化合物 $7 \mathbf{b}$ 对黄瓜灰霉病菌和水稻纹枯病菌的抑制率均为 $100 \%$. 进一步复篎后在 $50 \mathrm{mg} / \mathrm{L}$ 浓度下，化合物 7c、7d、7e、

$7 \mathrm{~g} 、 7 \mathrm{k} 、 71$ 对黄瓜灰霉病菌的抑制率均为 $62.06 \%$

表 3 目标化合物 $7 \mathbf{a} \sim 7 \mathbf{m}$ 的杀菌活性(抑制率 $/ \%)^{a}$

Table 3 Fungicidal activities (inhibition rate/\%) of compounds $7 \mathbf{a} \sim 7 \mathbf{m}$

\begin{tabular}{|c|c|c|c|c|c|c|}
\hline \multirow{2}{*}{ Compd. } & \multicolumn{3}{|c|}{ 黄瓜灰霉病菌(B. cinerea) } & \multicolumn{3}{|c|}{ 水稻纹枯病菌 $($ R. solani $)$} \\
\hline & $50 \mathrm{mg} \cdot \mathrm{L}^{-1}$ & $25 \mathrm{mg} \cdot \mathrm{L}^{-1}$ & $12.5 \mathrm{mg} \cdot \mathrm{L}^{-1}$ & $50 \mathrm{mg} \cdot \mathrm{L}^{-1}$ & $25 \mathrm{mg} \cdot \mathrm{L}^{-1}$ & $12.5 \mathrm{mg} \cdot \mathrm{L}^{-1}$ \\
\hline $7 a$ & 45.88 & 12.36 & - & 47.06 & 29.31 & 17.69 \\
\hline $7 \mathbf{b}$ & 52.06 & 41.18 & 30.08 & 51.77 & 41.71 & 31.77 \\
\hline $7 \mathrm{c}$ & 63.83 & 40.30 & 29.71 & 79.12 & 18.24 & 16.77 \\
\hline 7d & 62.06 & 49.41 & 32.94 & 50.00 & 42.06 & 20.06 \\
\hline $7 e$ & 66.47 & 14.71 & - & 57.90 & 18.83 & 11.77 \\
\hline $7 f$ & 42.06 & 14.41 & 6.77 & - & - & - \\
\hline $7 \mathrm{~g}$ & 65.00 & 44.71 & 17.36 & 66.77 & 36.77 & 6.18 \\
\hline $7 \mathrm{~h}$ & 50.59 & 11.76 & 0.88 & 68.83 & 8.82 & 1.47 \\
\hline $7 \mathbf{i}$ & 51.77 & 27.06 & 24.12 & - & - & - \\
\hline $7 \mathbf{j}$ & 41.76 & 7.65 & 3.83 & - & - & - \\
\hline $7 \mathrm{k}$ & 64.41 & 28.83 & 7.06 & 55.30 & 34.41 & 4.41 \\
\hline 71 & 67.35 & 38.21 & 29.71 & 49.12 & 25.30 & 19.12 \\
\hline $7 \mathrm{~m}$ & 47.65 & 30.30 & 9.71 & - & - & - \\
\hline 嘧菌酯 & 40 & 20 & 0 & 60 & 20 & 0 \\
\hline
\end{tabular}


表 4 部分目标化合物与嘧菌酯的杀菌活性(抑制率/\%)对照 ${ }^{a}$

Table 4 Comparison of fungicidal activities (inhibition rate/\%) of some compounds and azoxystrobin

\begin{tabular}{|c|c|c|c|c|c|c|}
\hline \multirow{2}{*}{ Compd. } & \multicolumn{3}{|c|}{ 黄瓜灰霉病菌(B. cinerea) } & \multicolumn{3}{|c|}{ 水稻纹枯病菌 $(R$. solani $)$} \\
\hline & $50 \mathrm{mg} \cdot \mathrm{L}^{-1}$ & $25 \mathrm{mg} \cdot \mathrm{L}^{-1}$ & $12.5 \mathrm{mg} \cdot \mathrm{L}^{-1}$ & $50 \mathrm{mg} \cdot \mathrm{L}^{-1}$ & $25 \mathrm{mg} \cdot \mathrm{L}^{-1}$ & $12.5 \mathrm{mg} \cdot \mathrm{L}^{-1}$ \\
\hline $7 \mathrm{c}$ & +23.83 & +20.30 & +29.71 & +19.12 & -1.76 & +16.77 \\
\hline $7 d$ & +22.06 & +29.41 & +32.94 & -10.00 & +22.06 & +20.06 \\
\hline $7 e$ & +26.47 & -5.29 & - & -2.10 & -1.17 & +11.77 \\
\hline $7 \mathrm{~g}$ & +25.00 & +24.71 & +17.36 & +6.77 & +16.77 & +6.18 \\
\hline $7 \mathrm{~h}$ & +10.59 & -8.24 & +0.88 & +8.83 & -11.18 & +1.47 \\
\hline $7 \mathbf{k}$ & +24.41 & +8.83 & +7.06 & -4.7 & +14.41 & +4.41 \\
\hline 71 & +27.35 & +18.21 & +29.71 & -10.88 & +5.30 & +19.12 \\
\hline 嘧菌酯 & 40 & 20 & 0 & 60 & 20 & 0 \\
\hline
\end{tabular}

$67.35 \%$, 活性均优于嘧菌酯; 化合物 $7 \mathrm{c} 、 7 \mathrm{~g} 、 7 \mathrm{~h}$ 对水稻 纹枯病菌的抑制率达 $66.77 \%$ 79.12\%, 活性优于嘧菌 酯, 值得进一步进行结构优化有望成为良好的杀菌剂先 导化合物.

\section{3 实验部分}

\section{1 仪器与试剂}

Bruker AVANC III $500 \mathrm{MHz}$ 核磁共振仪; 上海精密 科学仪器有限公司 X-4 型数字显示熔点测定仪(温度计 未校正); UPLC H-CLASS/QTOF G2-XS 质谱仪; 上海上 自仪转速表仪表电机有限公司 LOOYEZX98-1 旋转蒸发 仪; 柱层析分离纯化(柱层析硅胶为 $\mathrm{H}$ 型, 青岛海洋化 工厂, 200 300 目). 所用试剂和溶剂均为分析纯.

\section{2 中间体 $L-$ 丝氨酸甲酯盐酸盐(2)的合成}

参考文献[24], 在 $-10{ }^{\circ} \mathrm{C}$ 下将 $20 \mathrm{~mL}$ 氯化亚砜缓慢 滴入 $100 \mathrm{~mL}$ 无水甲醇, 控制流速 $30 \mathrm{~min}$ 滴完后, 继续 反应 $1 \mathrm{~h}$. 随后加入 $10.5 \mathrm{~g} L$-丝氨酸, 在 $38{ }^{\circ} \mathrm{C}$ 条件下反 应 $7 \mathrm{~h}$ 后反应结束, 加入 $20 \mathrm{~mL}$ 无水甲醇控制温度在 $40{ }^{\circ} \mathrm{C}$ 下进行抽滤, 用无水乙醚与石油醚进行重结晶, 最终得到 $8.7 \mathrm{~g}$ 白色固体. m.p. $157 \sim 158{ }^{\circ} \mathrm{C}$ (Lit. ${ }^{[24]}$ $\left.157.8 \sim 158.6{ }^{\circ} \mathrm{C}\right)$.

\section{3 中间体(4S)-2-(吡啶-2-基)-噁唑烷-4-羧酸甲酯和} (4R)-2-(吡啶-2-基)-噁唑烷-4-羧酸甲酯(3)的合成

参考文献 $[25,26]$, 向 $100 \mathrm{~mL}$ 圆底烧瓶中加入 $2.1 \mathrm{~g}$ (0.02 mol)吡啶-2-甲醛、 $3.1 \mathrm{~g}(0.02 \mathrm{~mol})$ 的 $L$-丝氨酸甲酯 盐酸盐、 $2.9 \mathrm{~g}(0.02 \mathrm{~mol})$ 无水硫酸钠、 $4.1 \mathrm{~g}(0.04 \mathrm{~mol})$ 三 乙胺和 $25 \mathrm{~mL}$ 二氯甲烷, 室温下搅拌反应 $10 \mathrm{~h}$, 薄层色 谱 $(\mathrm{TLC})[V$ (石油醚) $) V($ 乙酸乙酯 $)=1 ： 1]$ 监测反应. 反 应结束后, 反应液抽滤分离, 滤液旋蒸得到 $1.8 \mathrm{~g}$ 黄色油 状物, 粗收率 $83 \%$.

\section{4 中间体 2-(吡啶-2-基)-噁唑-4-甲酸甲酯(4)的合成} 参考文献[25], 向 $100 \mathrm{~mL}$ 圆底烧瓶中加入 $2.1 \mathrm{~g}$
(0.01 mol)中间体 3 和 $25 \mathrm{~mL}$ 二氯甲烷，在冰水浴下加入 $11.9 \mathrm{~g}(0.06 \mathrm{~mol})$ 三氯溴甲烷，搅拌下缓慢滴加 $9.1 \mathrm{~g}$ (0.06 mol) 1,8-二氮杂二环十一碳-7-烯(DBU), 反应 12 h. 结束反应后用柱层析 $[V$ (石油醚) $: V($ 乙酸乙酯 $)=1$ : 1]纯化得到 $1.4 \mathrm{~g}$ 淡黄色固体, 收率 68\%. m.p. 134 $135{ }^{\circ} \mathrm{C} ;{ }^{1} \mathrm{H}$ NMR $\left(500 \mathrm{MHz}, \mathrm{CDCl}_{3}\right) \delta: 8.77$ (d, $J=5.0$ $\mathrm{Hz}, 1 \mathrm{H}, \mathrm{Py}-\mathrm{H}), 8.41$ (s, 1H, CH), 8.31 (d, $J=8.0 \mathrm{~Hz}, 1 \mathrm{H}$, Py-H), 7.88 (t, $J=8.0 \mathrm{~Hz}, 1 \mathrm{H}, \mathrm{Py}-\mathrm{H}), 7.45$ (dd, $J=7.5,4.5$ $\mathrm{Hz}, 1 \mathrm{H}, \mathrm{Py}-\mathrm{H}), 3.98$ (s, 3H, $\left.\mathrm{CH}_{3}\right)$.

\section{5 中间体 2-(吡啶-2-基)-噁唑-4-甲酸(5)的合成}

向 $100 \mathrm{~mL}$ 圆底烧瓶中加入 $2.1 \mathrm{~g}(0.01 \mathrm{~mol})$ 中间体 4, 常温下用 $16 \mathrm{~mL}$ 甲醇水溶液 $\left[V(\mathrm{MeOH}): V\left(\mathrm{H}_{2} \mathrm{O}\right)=\right.$ $1: 1]$ 溶解, 再加入 $0.8 \mathrm{~g}(0.02 \mathrm{~mol})$ 氢氧化钠, 充分反应 $2 \mathrm{~h}$. 将反应液进行旋蒸后滴加盐酸, 析出固体, 减压抽 滤得到 $1.7 \mathrm{~g}$ 墨绿色固体, 收率 $92 \%$. m.p. $173 \sim 175{ }^{\circ} \mathrm{C}$.

\section{6 中间体 2-(吡啶-2-基)-噁唑-4-甲酰氯(6)的合成}

在干燥的 $50 \mathrm{~mL}$ 圆底烧瓶中加入 $0.4 \mathrm{~g}(2.0 \mathrm{mmol})$ 中间体 5 和 $20 \mathrm{~mL}$ 氯化亚砜, 加热回流反应 $2 \mathrm{~h}$, 蒸馏除 去过量氯化亚砜, 得到中间体 $\mathbf{6}$, 产品不经纯化直接用 于下一步反应.

\section{7 目标化合物 N-2-(2-吡啶基)-噁唑酰胺类化合物 $7 \mathrm{a} \sim 7 \mathrm{~m}$ 的制备}

在 $100 \mathrm{~mL}$ 圆底烧瓶加入 $3.0 \mathrm{mmol}$ 对应含取代基的 苯胺、 $0.6 \mathrm{~g}$ 三乙胺和 $20 \mathrm{~mL}$ 二氯甲烷, 再缓慢加入中间 体 6, 冰浴反应 $2 \mathrm{~h}$. 反应液抽滤除去溶剂, 再经过柱层 析 $[V($ 石油醚 $): V($ 乙酸乙酯 $)=1: 1]$ 分离得到目标化合 物 $7 \mathbf{a} \sim 7 \mathrm{~m}$.

$\mathrm{N}$-2-氟苯基-2-(吡啶-2-基)-噁唑-4-甲酰胺 (7a)：淡 黄色固体, 收率 $81 \%$. m.p. $123 \sim 124{ }^{\circ} \mathrm{C} ;{ }^{1} \mathrm{H}$ NMR $(600$ MHz, DMSO- $\left.d_{6}\right) \delta: 9.92(\mathrm{~s}, 1 \mathrm{H}, \mathrm{NH}), 8.98(\mathrm{~s}, 1 \mathrm{H}$, oxazole-H), 8.77 (d, $J=4.8 \mathrm{~Hz}, 1 \mathrm{H}$, Py-H), 8.22 (d, $J=7.8$ $\mathrm{Hz}, 1 \mathrm{H}, \mathrm{Py}-\mathrm{H}), 8.04 \sim 8.07$ (m, 1H, Py-H), $7.81 \sim 7.82(\mathrm{~m}$, $1 \mathrm{H}$, Ar-H), $7.62 \sim 7.63(\mathrm{~m}, 1 \mathrm{H}, \mathrm{Py}-\mathrm{H}), 7.34 \sim 7.35(\mathrm{~m}, 1 \mathrm{H}$, 
Ar-H), $7.27 \sim 7.28(\mathrm{~m}, 2 \mathrm{H}, \mathrm{Ar}-\mathrm{H}) ;{ }^{13} \mathrm{C}$ NMR $(151 \mathrm{MHz}$, DMSO- $\left.d_{6}\right) \delta: 160.4,158.9,156.3,154.6,150.6,145.1$, 144.5, 138.2, 137.3, 127.2 (d, $J=7.6 \mathrm{~Hz}), 126.3$ (d, $J=$ $26.0 \mathrm{~Hz}), 125.5(\mathrm{~d}, J=11.9 \mathrm{~Hz}), 125.0(\mathrm{~d}, J=3.3 \mathrm{~Hz})$, 123.1, $116.2(\mathrm{~d}, J=19.6 \mathrm{~Hz})$; HRMS calcd for $\mathrm{C}_{15} \mathrm{H}_{11} \mathrm{~F}-$ $\mathrm{N}_{3} \mathrm{O}_{2}[\mathrm{M}+\mathrm{H}]^{+}$284.0830, found 284.0822.

$N$-4-氟苯基-2-(吡啶-2-基)-噁唑-4-甲酰胺 (7b)：淡 黄色固体, 收率 77\%. m.p. $147 \sim 149{ }^{\circ} \mathrm{C} ;{ }^{1} \mathrm{H}$ NMR $(600$ MHz, DMSO- $\left.d_{6}\right) \delta: 10.32(\mathrm{~s}, 1 \mathrm{H}, \mathrm{NH}), 8.95$ (s, $1 \mathrm{H}$, oxazole-H), 8.77 (d, $J=4.8 \mathrm{~Hz}, 1 \mathrm{H}, \mathrm{Py}-\mathrm{H}), 8.23$ (d, $J=8.0$ $\mathrm{Hz}, 1 \mathrm{H}, \mathrm{Py}-\mathrm{H}), 8.07$ (td, $J=8.0,1.5 \mathrm{~Hz}, 1 \mathrm{H}, \mathrm{Py}-\mathrm{H}), 7.84 \sim$ 7.87 (m, 2H, Ar-H), $7.61 \sim 7.62(\mathrm{~m}, 1 \mathrm{H}$, Py-H), 7.21 (t, $J=$ $9.0 \mathrm{~Hz}, 2 \mathrm{H}, \mathrm{Ar}-\mathrm{H}) ;{ }^{13} \mathrm{C}$ NMR (151 MHz, DMSO- $\left.d_{6}\right) \delta$ : $160.3,159.8,159.0,158.2,150.6,145.3,144.4,138.2$, 137.9, 135.1, 126.3, 123.0 (t, $J=123.1,123.0,122.9 \mathrm{~Hz}$ ), $115.7(\mathrm{~d}, J=22.1 \mathrm{~Hz})$; HRMS calcd for $\mathrm{C}_{15} \mathrm{H}_{11} \mathrm{FN}_{3} \mathrm{O}_{2}$ $[\mathrm{M}+\mathrm{H}]^{+}$284.0830, found 284.0820.

$N$-2,4-二氟苯基-2-(吡啶-2-基)-噁唑-4-甲酰胺(7c): 淡黄色固体, 收率 76\%. m.p. $137 \sim 139{ }^{\circ} \mathrm{C} ;{ }^{1} \mathrm{H}$ NMR $\left(500 \mathrm{MHz}, \mathrm{DMSO}-d_{6}\right) \delta: 10.06(\mathrm{~s}, 1 \mathrm{H}, \mathrm{NH}), 8.97(\mathrm{~s}, 1 \mathrm{H}$, oxazole-H), 8.77 (d, $J=5.0,1 \mathrm{H}, \mathrm{Py}-\mathrm{H}), 8.21$ (d, $J=8.0$ Hz, 1H, Py-H), 8.06 8.07 (m, 1H, Py-H), 7.71 7.72 (m, $1 \mathrm{H}, \operatorname{Ar}-\mathrm{H}), 7.61(\mathrm{q}, J=3.0 \mathrm{~Hz}, 1 \mathrm{H}, \mathrm{Py}-\mathrm{H}), 7.39 \sim 7.40(\mathrm{~m}$, 1H, Ar-H), 7.14 (t, $J=4.5 \mathrm{~Hz}, 1 \mathrm{H}, \mathrm{Ar}-\mathrm{H}) ;{ }^{13} \mathrm{C}$ NMR $(126$ MHz, DMSO- $\left.d_{6}\right) \delta: 159.9,158.6,150.1,144.7,144.0$, 137.7, 136.7, 127.6 (d, $J=11.0 \mathrm{~Hz}), 125.8,122.6,111.3$ (d, $J=3.4 \mathrm{~Hz}), 111.2(\mathrm{~d}, J=3.4 \mathrm{~Hz}), 104.5,104.3(\mathrm{~d}, J=2.8$ $\mathrm{Hz})$, 104.1; HRMS calcd for $\mathrm{C}_{15} \mathrm{H}_{10} \mathrm{~F}_{2} \mathrm{~N}_{3} \mathrm{O}_{2}[\mathrm{M}+\mathrm{H}]^{+}$ 302.0736, found 302.0731 .

$N$-2,6-二氟苯基-2-(吡啶-2-基)-噁唑-4-甲酰胺(7d): 淡黄色固体, 收率 74\%. m.p. $127 \sim 129{ }^{\circ} \mathrm{C} ;{ }^{1} \mathrm{H}$ NMR $\left(600 \mathrm{MHz}, \mathrm{DMSO}-d_{6}\right) \delta: 10.03(\mathrm{~s}, 1 \mathrm{H}, \mathrm{NH}), 8.97(\mathrm{~s}, 1 \mathrm{H}$, oxazole-H), $8.77 \sim 8.79(\mathrm{~m}, 1 \mathrm{H}, \mathrm{Py}-\mathrm{H}), 8.21(\mathrm{~d}, J=7.8 \mathrm{~Hz}$, $1 \mathrm{H}, \mathrm{Py}-\mathrm{H}), 8.06 \sim 8.07(\mathrm{~m}, 1 \mathrm{H}, \mathrm{Py}-\mathrm{H}), 7.72 \sim 7.73(\mathrm{~m}, 1 \mathrm{H}$, Ar-H), $7.61 \sim 7.63(\mathrm{~m}, 1 \mathrm{H}$, Py-H), $7.39 \sim 7.41(\mathrm{~m}, 1 \mathrm{H}$, Ar-H), $7.15 \sim 7.16(\mathrm{~m}, 1 \mathrm{H}, \mathrm{Ar}-\mathrm{H}) ;{ }^{13} \mathrm{C}$ NMR $(151 \mathrm{MHz}$, DMSO- $\left.d_{6}\right) \delta: 160.4,159.1,150.6,145.2,144.5,138.2$, $137.2,128.2(\mathrm{~d}, J=9.5 \mathrm{~Hz}), 126.3,123.1,111.7$ (dd, $J=$ 22.1, $3.5 \mathrm{~Hz}$ ), 104.8 (dd, $J=26.6,24.2 \mathrm{~Hz}$ ); HRMS calcd for $\mathrm{C}_{15} \mathrm{H}_{10} \mathrm{~F}_{2} \mathrm{~N}_{3} \mathrm{O}_{2}[\mathrm{M}+\mathrm{H}]^{+}$302.0736, found 302.0726.

$N$-3-氯苯基-2-(吡啶-2-基)-噁唑-4-甲酰胺 (7e): 淡 黄色固体, 收率 80\%. m.p. $121 \sim 122{ }^{\circ} \mathrm{C} ;{ }^{1} \mathrm{H}$ NMR $(600$ MHz, DMSO- $\left.d_{6}\right) \delta: 10.44(\mathrm{~s}, 1 \mathrm{H}, \mathrm{NH}), 8.98(\mathrm{~s}, 1 \mathrm{H}$, oxazole-H), 8.77 (d, $J=4.8 \mathrm{~Hz}, 1 \mathrm{H}, \mathrm{Py}-\mathrm{H}), 8.24$ (d, $J=7.8$ Hz, 1H, Py-H), $8.07 \sim 8.08$ (m, 1H, Py-H), $8.03 \sim 8.04$ (m,
1H, Ar-H), 7.80 (d, J=8.2, $2.4 \mathrm{~Hz}, 1 \mathrm{H}, \mathrm{Ar}-\mathrm{H}), 7.62 \sim 7.63$ (m, 1H, Py-H), 7.40 (t, $J=8.2 \mathrm{~Hz}, 1 \mathrm{H}, \mathrm{Ar}-\mathrm{H}), 7.19 \sim 7.20$ (m, $1 \mathrm{H}, \mathrm{Ar}-\mathrm{H}) ;{ }^{13} \mathrm{C}$ NMR (126 MHz, DMSO) $\delta: 159.8$, $158.8,150.1,144.7,144.2,139.8,137.7,137.2,132.9$, 130.3, 125.8, 123.0, 122.6, 120.0, 118.9; HRMS calcd for $\mathrm{C}_{15} \mathrm{H}_{11} \mathrm{ClN}_{3} \mathrm{O}_{2}[\mathrm{M}+\mathrm{H}]^{+}$300.0534, found 300.0522.

$N$-3,5-二氯苯基-2-(吡啶-2-基)-噁唑-4-甲酰胺(7f): 淡黄色固体, 收率 $72 \%$. m.p. $112 \sim 113{ }^{\circ} \mathrm{C} ;{ }^{1} \mathrm{H}$ NMR $\left(600 \mathrm{MHz}, \mathrm{DMSO}-d_{6}\right) \delta: 10.61(\mathrm{~s}, 1 \mathrm{H}, \mathrm{NH}), 9.00(\mathrm{~s}, 1 \mathrm{H}$, oxazole-H), 8.77 (d, $J=4.6 \mathrm{~Hz}, 1 \mathrm{H}, \mathrm{Py}-\mathrm{H}), 8.23$ (d, $J=$ $7.8 \mathrm{~Hz}, 1 \mathrm{H}$, Py-H), $8.07 \sim 8.08$ (m, 1H, Py-H), 7.99 (d, $J=$ $1.8 \mathrm{~Hz}, 2 \mathrm{H}, \mathrm{Ar}-\mathrm{H}), 7.62 \sim 7.63(\mathrm{~m}, 1 \mathrm{H}, \mathrm{Py}-\mathrm{H}), 7.36(\mathrm{t}, J=$ $1.8 \mathrm{~Hz}, 1 \mathrm{H}, \mathrm{Ar}-\mathrm{H}) ;{ }^{13} \mathrm{C}$ NMR (151 MHz, DMSO- $\left.d_{6}\right) \delta$ : $160.4,159.5,150.6,145.1,141.2,140.7,138.2,137.4$, 134.4, 126.4, 123.7, 123.1, 119.1; HRMS calcd for $\mathrm{C}_{15} \mathrm{H}_{10} \mathrm{Cl}_{2} \mathrm{~N}_{3} \mathrm{O}_{2}[\mathrm{M}+\mathrm{H}]^{+}$334.0145, found334.0135.

$\mathrm{N}$-3-氯-2-甲基苯基-2-(吡啶-2-基)-噁唑-4-甲酰胺 (7g): 淡黄色固体，收率 77\%. m.p. 132 133 ${ }^{\circ} \mathrm{C} ;{ }^{1} \mathrm{H}$ NMR (500 MHz, DMSO- $\left.d_{6}\right) \delta: 10.15$ (s, $\left.1 \mathrm{H}, \mathrm{NH}\right), 8.95$ (s, $1 \mathrm{H}$, oxazole-H), 8.77 (d, J=5.0 Hz, 1H, Py-H), 8.22 (d, $J=7.8 \mathrm{~Hz}, 1 \mathrm{H}, \mathrm{Py}-\mathrm{H}), 8.07 \sim 8.08(\mathrm{~m}, 1 \mathrm{H}, \mathrm{Py}-\mathrm{H}), 7.62 \sim$ 7.63 (m, 1H, Py-H), 7.43 (dd, J=7.8, $1.2 \mathrm{~Hz}, 1 \mathrm{H}$, Ar-H), $7.38 \sim 7.39(\mathrm{~m}, 1 \mathrm{H}, \mathrm{Ar}-\mathrm{H}), 7.26(\mathrm{t}, J=7.8 \mathrm{~Hz}, 1 \mathrm{H}, \mathrm{Ar}-\mathrm{H})$, $2.28\left(\mathrm{~s}, 3 \mathrm{H}, \mathrm{CH}_{3}\right) ;{ }^{13} \mathrm{C}$ NMR (126 MHz, DMSO) $\delta: 160.3$, $159.1,150.6,145.2,144.4,138.2,137.6,137.6,134.2$, $132.0,127.4,127.2,126.3,125.7,123.0,15.7$; HRMS calcd for $\mathrm{C}_{16} \mathrm{H}_{13} \mathrm{ClN}_{3} \mathrm{O}_{2}[\mathrm{M}+\mathrm{H}]^{+}$314.0691, found 314.0690 .

$N$-3-溴苯基-2-(吡啶-2-基)-噁唑-4-甲酰胺(7h)：淡 黄色固体, 收率 70\%. m.p. $161 \sim 162{ }^{\circ} \mathrm{C} ;{ }^{1} \mathrm{H}$ NMR $(600$ MHz, DMSO- $\left.d_{6}\right) \delta: 10.42(\mathrm{~s}, 1 \mathrm{H}, \mathrm{NH}), 8.97$ (s, $1 \mathrm{H}$, oxazole-H), 8.77 (d, $J=4.2 \mathrm{~Hz}, 1 \mathrm{H}, \mathrm{Py}-\mathrm{H}), 8.24$ (d, $J=7.8$ $\mathrm{Hz}, 1 \mathrm{H}, \mathrm{Py}-\mathrm{H}), 8.16$ (d, $J=1.8 \mathrm{~Hz}, 1 \mathrm{H}, \mathrm{Ar}-\mathrm{H}), 8.07 \sim 8.08$ (m, 1H, Py-H), 7.84 (dt, $J=7.4,1.8 \mathrm{~Hz}, 1 \mathrm{H}, \mathrm{Ar}-\mathrm{H}), 7.62 \sim$ 7.64 (m, 1H, Py-H), 7.34 7.35 (m, 2H, Ar-H); ${ }^{13} \mathrm{C}$ NMR (126 MHz, DMSO) $\delta: 159.8,158.8,150.1,144.7,144.2$, $139.9,137.7,137.2,130.6,126.6,125.8,122.8,122.6$, 121.4, 119.3; HRMS calcd for $\mathrm{C}_{15} \mathrm{H}_{11} \mathrm{BrN}_{3} \mathrm{O}_{2}[\mathrm{M}+\mathrm{H}]^{+}$ 344.0029 , found 344.0033 .

$N$-3-三氟甲基苯基-2-(吡啶-2-基)-噁唑-4-甲酰胺 (7i): 淡黄色固体, 收率 71\%. m.p. $133 \sim 134{ }^{\circ} \mathrm{C} ;{ }^{1} \mathrm{H}$ NMR $\left(600 \mathrm{MHz}\right.$, DMSO- $\left.d_{6}\right) \delta: 10.60(\mathrm{~s}, 1 \mathrm{H}, \mathrm{NH}), 8.99$ (t, $J=3.2 \mathrm{~Hz}, 1 \mathrm{H}$, oxazole-H), $8.77 \sim 8.78(\mathrm{~m}, 1 \mathrm{H}, \mathrm{Py}-\mathrm{H})$, $8.31 \sim 8.32$ (m, 1H, Ar-H), 8.24 8.25 (m, 1H, Py-H), 8.14 $(\mathrm{d}, J=8.2 \mathrm{~Hz}, 1 \mathrm{H}, \mathrm{Ar}-\mathrm{H}), 8.08 \sim 8.09(\mathrm{~m}, 1 \mathrm{H}, \mathrm{Py}-\mathrm{H}), 7.62$ 
(t, $J=7.0 \mathrm{~Hz}, 2 \mathrm{H}, \mathrm{Py}-\mathrm{H}), 7.49$ (d, $J=7.6 \mathrm{~Hz}, 1 \mathrm{H}, \mathrm{Ar}-\mathrm{H})$;

${ }^{13} \mathrm{C}$ NMR $\left(126 \mathrm{MHz}\right.$, DMSO- $\left.d_{6}\right) \delta: 159.9,159.0,150.1$, 144.7, 144.3, 139.1, 137.7, 137.1, 129.8, 129.4 (d, $J=31.7$ $\mathrm{Hz}), 125.8,124.1,122.6,120.3$ (d, $J=4.0 \mathrm{~Hz}), 116.7$ (d, $J=4.0 \mathrm{~Hz}$ ); HRMS calcd for $\mathrm{C}_{16} \mathrm{H}_{11} \mathrm{~F}_{3} \mathrm{~N}_{3} \mathrm{O}_{2}[\mathrm{M}+\mathrm{H}]^{+}$ 334.0798, found 334.0794.

$N$-4-甲基苯基-2-(吡啶-2-基)-噁唑-4-甲酰胺(7j) : 淡 黄色固体, 收率 $80 \%$. m.p. $149 \sim 150{ }^{\circ} \mathrm{C} ;{ }^{1} \mathrm{H}$ NMR $(600$ MHz, DMSO- $\left.d_{6}\right) \delta: 10.12(\mathrm{~s}, 1 \mathrm{H}, \mathrm{NH}), 8.92(\mathrm{~s}, 1 \mathrm{H}$, oxazole-H), 8.76 (d, $J=4.2 \mathrm{~Hz}, 1 \mathrm{H}, \mathrm{Py}-\mathrm{H}), 8.24$ (d, $J=7.8$ Hz, 1H, Py-H), 8.06 8.07 (m, 1H, Py-H), 7.70 (d, $J=8.4$ $\mathrm{Hz}, 2 \mathrm{H}$, Ar-H), $7.60 \sim 7.61(\mathrm{~m}, 1 \mathrm{H}, \mathrm{Py}-\mathrm{H}), 7.17$ (d, $J=$ $17.2 \mathrm{~Hz}, 2 \mathrm{H}, \mathrm{Ar}-\mathrm{H}), 2.29$ (s, 3H, $\left.\mathrm{CH}_{3}\right) ;{ }^{13} \mathrm{C}$ NMR $(151$ MHz, DMSO- $\left.d_{6}\right) \delta: 160.2,158.8,150.6,145.3,144.2$, $138.2,138.0,136.2,133.5,129.5,126.3,123.1,121.0$, 21.0; HRMS calcd for $\mathrm{C}_{16} \mathrm{H}_{14} \mathrm{~N}_{3} \mathrm{O}_{2}[\mathrm{M}+\mathrm{H}]^{+}$280.1081, found 280.1065 .

$N$-2,4-二甲基苯基-2-(吡啶-2-基)-噁唑-4-甲酰胺 (7k): 淡黄色固体, 收率 78\%. m.p. $155 \sim 157{ }^{\circ} \mathrm{C} ;{ }^{1} \mathrm{H}$ NMR (500 MHz, DMSO- $\left.d_{6}\right) \delta$ : 9.73 (s, 1H, NH), 8.91 (s, $1 \mathrm{H}$, oxazole-H), $8.76 \sim 8.78$ (m, 1H, Py-H), 8.22 (d, $J=8.0$ Hz, 1H, Py-H), 8.06 8.08 (m, 1H, Py-H), $7.61 \sim 7.63$ (m, 1H, Py-H), $7.36 \sim 7.37$ (m, $1 \mathrm{H}, \mathrm{Ar}-\mathrm{H}), 7.10(\mathrm{~d}, J=2.0 \mathrm{~Hz}$, 1H, Ar-H), 7.03 (d, J=8.0 Hz, 1H, Ar-H), 2.29 (s, 3H, $\left.\mathrm{CH}_{3}\right), 2.23$ (s, 3H, $\left.\mathrm{CH}_{3}\right) ;{ }^{13} \mathrm{C} \mathrm{NMR}\left(126 \mathrm{MHz}, \mathrm{CDCl}_{3}\right) \delta$ : $159.8,158.3,150.1,144.8,143.4,137.6,137.4,134.9$, $132.9,132.2,130.9,126.6,125.7,125.3,20.5,17.6$; HRMS calcd for $\mathrm{C}_{17} \mathrm{H}_{16} \mathrm{~N}_{3} \mathrm{O}_{2}[\mathrm{M}+\mathrm{H}]^{+}$294.1237, found 294.1226.

$\mathrm{N}$-4-乙氧基苯基-2-(吡啶-2-基)-噁唑-4-甲酰胺(7l): 淡黄色固体, 收率 $69 \%$. m.p. $154 \sim 155{ }^{\circ} \mathrm{C} ;{ }^{1} \mathrm{H}$ NMR $\left(600 \mathrm{MHz}, \mathrm{DMSO}-d_{6}\right) \delta: 10.09$ (s, $\left.1 \mathrm{H}, \mathrm{NH}\right), 8.91(\mathrm{~s}, 1 \mathrm{H}$, oxazole-H), 8.76 (d, $J=4.5 \mathrm{~Hz}, 1 \mathrm{H}, \mathrm{Py}-\mathrm{H}), 8.23$ (d, $J=7.8$ Hz, 1H, Py-H), 8.06 8.09 (m, 1H, Py-H), 7.71 (d, $J=9.0$ $\mathrm{Hz}, 2 \mathrm{H}, \mathrm{Ar}-\mathrm{H}), 7.60 \sim 7.61$ (m, 1H, Py-H), 6.93 (d, $J=9.0$ $\mathrm{Hz}, 2 \mathrm{H}, \mathrm{Ar}-\mathrm{H}), 4.02$ (q, $J=7.0 \mathrm{~Hz}, 2 \mathrm{H}, \mathrm{CH}_{2}$ ), 1.33 (t, $J=$ $\left.7.0 \mathrm{~Hz}, 3 \mathrm{H}, \mathrm{CH}_{3}\right) ;{ }^{13} \mathrm{C}$ NMR $\left(151 \mathrm{MHz}\right.$, DMSO- $\left.d_{6}\right) \delta$ : $160.2,158.6,155.5,150.6,145.3,144.1,138.2,138.1$, 126.3, 123.1, 122.6, 114.8, 63.6, 15.2; HRMS calcd for $\mathrm{C}_{17} \mathrm{H}_{16} \mathrm{~N}_{3} \mathrm{O}_{3}[\mathrm{M}+\mathrm{H}]^{+}$310.1186, found 310.1174 .

$N$-4-叔丁基苯基-2-(吡啶-2-基)-噁唑-4-甲酰胺(7m): 淡黄色固体, 收率 67\%. m.p. $14 \sim 143{ }^{\circ} \mathrm{C} ;{ }^{1} \mathrm{H}$ NMR $(600$ MHz, DMSO- $\left.d_{6}\right) \delta: 10.13$ (s, $\left.1 \mathrm{H}, \mathrm{NH}\right), 8.93$ (s, $1 \mathrm{H}$, oxazole-H), 8.76 (d, $J=4.8 \mathrm{~Hz}, 1 \mathrm{H}, \mathrm{Py}-\mathrm{H}), 8.24$ (d, $J=7.8$ Hz, 1H, Py-H), $8.06 \sim 8.08$ (m, 1H, Py-H), 7.73 (d, $J=8.8$
Hz, 2H, Ar-H), 7.61 (q, $J=7.2,1 \mathrm{H}$, Py-H), 7.38 (d, $J=8.4$ $\mathrm{Hz}, 2 \mathrm{H}, \mathrm{Ar}-\mathrm{H}), 1.29$ (s, 9H, $\left.\left(\mathrm{CH}_{3}\right)_{3}\right) ;{ }^{13} \mathrm{C}$ NMR $(151 \mathrm{MHz}$, DMSO- $\left.d_{6}\right) \delta$ : $160.2,158.8,150.6,146.9,145.3,144.2$, 138.2, 138.0, 136.1, 126.3, 125.8, 123.1, 120.9, 34.6, 31.7; HRMS calcd for $\mathrm{C}_{19} \mathrm{H}_{20} \mathrm{~N}_{3} \mathrm{O}_{2}[\mathrm{M}+\mathrm{H}]^{+} 322.1550$, found 322.1540 .

\section{8 杀菌活性测试}

实验方法: 将溶化好的马铃薯葡萄糖琼脂培养基 (PDA) 冷却至 $60 \sim 70{ }^{\circ} \mathrm{C}$, 按设计浓度加入定量药剂, 配制成含有不同药量的含毒培养基. 待其充分冷却 后，接种直径为 $5 \mathrm{~mm}$ 的香蕉叶斑病菌的菌饼，然后放 置于培养箱中培养. 按上述方法, 每个浓度做 3 组重 复实验，并设立一组空白对照组. 在 $96 \mathrm{~h}$ 后(不同的 病菌, 调查时间不同), 采用十字交叉法进行结果调 查.

药效计算：分别测量每个样品的菌落直径, 然后 计算抑菌率.

辅助材料(Supporting Information) 化合物 $7 \mathrm{a} \sim 7 \mathrm{~m}$ 的 ${ }^{1} \mathrm{H}$ NMR, ${ }^{13} \mathrm{C}$ NMR 和 EI-MS 谱图. 这些材料可以免费 从本刊网站(http://sioc-journal.cn/)上下载.

\section{References}

[1] Yang, J. C.; Guan, A. Y.; Yang, F.; Liu, C. L. Modern Agrochem. 2015, 14, 1 (in Chinese). (杨吉春，关爱莹，杨帆，刘长令，现代农药, 2015, 14, 1.)

[2] Wu, Z. B.; Kuang, J. Q.; Yin, J.; Wu, S. X.; Cai, H. Guizhou Agric. Sci. 2013, 41, 93 (in Chinese). (吴志兵，广继清，尹娟，吴世喜，蔡桦，贵州农业科学，2013， 41,93.)

[3] Kaspady, M.; Narayanaswamy, V. K.; Raju, M.; Rao, G. K. Lett. Drug Des. Discovery 2009, 6, 21.

[4] Li, Y.; Zhang, H. Q.; Liu, J.; Yang, X. P.; Liu, Z. J. J. Agric. Food Chem. 2006, 54, 3636.

[5] Dai, H.; Zhuang. H. Y.; Shi, L.; Li, G.; Zhang, H. J.; Fang, Y.; Dai, B. J. Chin. J. Org. Chem. 2015, 35, 2399 (in Chinese). (戴红, 庄辉阳, 施否, 李刚, 张海军, 方源, 戴宝江, 有机化学, 2015, 35, 2399.)

[6] Dai, H.; Li, Y. Q.; Du, D.; Qin, X.; Zhang, X.; Yu, H. B.; Fang, J. X J. Agric. Food Chem. 2008, 56, 10805.

[7] Dai, H.; Li, H.; Jin, Z. C.; Liu, W. Y.; Xiao, Y.; He, H. B.; Wang, Q. M.; Shi, Y. J. Chin. J. Org. Chem. 2016, 36, 185 (in Chinese). (戴红, 李宏, 金智超, 刘文永, 肖瑶, 何海兵, 汪清民, 石玉军, 有机化学, 2016, 36, 185.)

[8] Yang, Y. G.; Meng, S. Q.; Qi, Z. Q.; Ji, M. S.; Li, X. H. Chin. J. Pestic. Sci. 2018, 20, 287 (in Chinese). (杨永贵，孟司奇，祁之秋，纪明山，李兴海，农药学学报，2018， 20, 287.)

[9] Wu, J.; Song, B. A.; Kang, S. D.; Yang, S.; Hu, D. Y. Pesticides 2012, 51, 625 (in Chinese). (吴剑，宋宝安，康圣鸿，杨松 胡德禹，农药, 2012, 51, 625.)

[10] Liu, X. H.; Zhao, W.; Shen, Z. H.; Xing, J. H.; Yuan, J.; Yang, G.; Xu, T. M.; Peng, W. L. Bioorg. Med. Chem. Lett. 2016, 26, 3626.

[11] Liu, X. H.; Zhao, W.; Shen, Z. H.; Xing, J. H.; Xu, T. M.; Peng, W. L. Eur. J. Med. Chem. 2017, 125, 881.

[12] Lahm, G. P.; Cordova, D.; Barry, J. D. Bioorg. Med. Chem. 2009, 
$17,4127$.

[13] Ji, W. J.; Xu, T. M.; Zheng, Z. W.; Zhu, B. C.; Li, J.; Hu, W. Q.; Kong, X. L. Chin. J. Pestic. Sci. 2013, 15, 393 (in Chinese). (姬文娟, 许天明, 郑志文, 朱冰春, 李姣, 胡伟群, 孔小林, 农 药学学报, 2013, 15, 393.)

[14] Eryılmaz, S.; Çelikoğlu, E. T.; İdil, Ö.; İnkaya, E.; Kozak, Z.; Mısır, E.; Gül, M. Bioorg. Chem. 2020, 95, 103476.

[15] Zhong, B.; Liu, C. L.; Zhao, W. G.; Li, Z. M. Chin. J. Org. Chem. 2004, 24, 204 (in Chinese). (钟滨, 刘长令, 赵卫光, 李正名, 有机化学, 2004, 24, 204.)

[16] Ouyang, G. P.; Cai, X. J.; Chen, Z.; Song, B. A.; Bhadury, P. S.; Yang, S.; Jin, L. H.; Xue, W.; Hu, D. Y.; Zeng, S. J. Agric. Food Chem. 2008, 56, 10160 .

[17] Wang, X.; Wang, C. Q.; Fu, C. R.; Zou, X. M. Chin. J. Org. Chem. 2015, 35, 92 (in Chinese)

(王金金，王朝强，傅翠蓉，邹小毛，有机化学， 2015, 35, 92.)

[18] Wang, M. M.; Zhang, Q. Q.; Yue, K.; Li, Q. S.; Xu, F. B. Chin. J. Org. Chem. 2017, 37, 1774 (in Chinese).

(王梦梦, 张青青, 岳凯, 李庆山, 徐风波, 有机化学, 2017, 37, 1774.)

[19] Gideens, A. C.; Boshoff, H. I. M.; Franzblau, S. G.; Barry III, C. E.;
Coppa, B. R. Tetrahedron Lett. 2005, 46, 7355.

[20] Prakash, T. B.; Reddy, G. D.; Padmaja, A.; Padmavathi, V. Eur. J. Med. Chem. 2014, 82, 347.

[21] Lin, J.; Chen, J. W.; Cai, X. Y.; Qiao, X. L.; Huang, L. P.; Wang, D. G.; Wang, Z. J. Agric. Food Chem. 2007, 55, 7626

[22] Shi, Y. J.; Du, X. C.; Wang, X. L.; Chen, Q. W.; Li, L.; Dai, H.; Xu, C. Q.; Zhang, J, Y.; Ling, Y. Chin. J. Org. Chem. 2018, 38, 1772 (in Chinese).

(石玉军, 杜显超，王祥龙，陈庆文，李玲，戴红，徐蔡芹，张敬 远, 凌勇, 有机化学, 2018, 38, 1772.)

[23] Liu, T. T.; Ni, Y.; Zhong, L. K.; Huang, H. Y.; Hu, W. Q.; Xu, T. M.; Tan, C. X. Chin. J. Org. Chem. 2015, 35, 422 (in Chinese). (刘婷婷，倪芸，钟良坤，黄红英，胡伟群，许天明，谭成侠，有 机化学, 2015, 35, 422.)

[24] Ji, J.; Lu, E. B.; Wan, W. T.; Li, M.; Lei, H.; Ding, Y. G.; Xiong, J. L. Chem. Bioeng. 2008, 25, 51 (in Chinese).

(喊 峻, 鲁尔贝, 万雯婷, 黎明, 雷吴, 丁一刚, 熊家林, 化学与 生物工程, 2008, 25, 51.)

[25] Graham, T. H. Org. Lett. 2010, 12, 3614

[26] Credico, B. D.;Reginato, G.; Gonsalvi, L.; Peruzzini, M.; Rossin, A. Tetrahedron 2011, 67, 267. 